\title{
A Study of Clinical and Serological Correlation of Positive Direct Antiglobulin Test in Blood Bank at a Tertiary Care Center
}

\author{
Alisha Suresh Kerkar ${ }^{1}$ Swarupa Nikhil Bhagwat ${ }^{1}$ Jayashree Harihara Sharma ${ }^{1}$ \\ ${ }^{1}$ Department of Transfusion Medicine, Seth GS Medical College and \\ KEM Hospital, Mumbai, Maharashtra, India \\ J Lab Physicians 2022;14:223-230. \\ Address for correspondence Swarupa Nikhil Bhagwat, MD, \\ Department of Transfusion Medicine, Seth GS Medical College and \\ KEM Hospital, Mumbai 400012, India \\ (e-mail: swarupabhagwat@kem.edu).
}

\begin{abstract}
Keywords

- direct antiglobulin test

- in vivo hemolysis

- autoimmune hemolytic anemia

- connective tissue disorders
\end{abstract}

Objectives Detection of red cell bound immunoglobulins and/or complement by direct antiglobulin test (DAT) is a crucial serological assay in the diagnosis of autoimmune hemolytic anemia (AIHA). However, DAT may be positive in a variety of clinical conditions with or without hemolysis. We aimed at evaluating the clinical and serological correlation of positive DAT by categorizing the clinical conditions associated with positive DAT, estimating the presence of in vivo hemolysis in case of positive DAT with polyspecific and monospecific antisera and correlating the strength of positive DAT with the presence of hemolysis.

Materials and Methods The prospective observational study was performed on 200 samples that were positive for DAT with polyspecific antiglobulin reagent as the baseline investigation. These samples were further tested with anti-immunoglobulin $\mathrm{G}$ and anti-C3 monospecific DAT reagents to evaluate the type of protein responsible for positive DAT. The antiglobulin tests were performed by tube technique. DAT positivity was graded $(1+$ to $4+)$ in each patient. Autocontrol test was included. The patients with positive polyspecific DAT were categorized into different clinical conditions. The presence or absence of in vivo hemolysis was evaluated in all clinical categories and also for each grade of positivity with polyspecific and monospecific antiglobulin reagents. Statistical Analysis Binomial logistic regression and Mann-Whitney $U$ test were applied to between the group analyses. For categorical variables, Fisher's exact test and relative risk were used. The qualitative data were expressed in numbers and percentages.

Results The highest number of patients (75/200, 37.5\%) belonged to the autoimmune diseases group. Tuberculosis and hepatitis $C$ were the main infectious diseases associated with positive DAT. Out of 200 DAT-positive patients, 98 (49\%) had in vivo hemolysis and 102 (51\%) did not have hemolysis. AlHA (22) and systemic lupus erythematosus (18) were the commonest clinical conditions associated with in vivo hemolysis. All the 11 samples that showed positivity with only anti-C3 reagent did not show any hemolysis. There was statistically significant increase in the incidence of in vivo hemolysis with increasing grades of DAT positivity with all the three antihuman globulin reagents.

Conclusion There are different disease conditions which show positive DAT with or without hemolysis. So, it is important to clinically and serologically correlate positive DAT results. published online January 18, 2022
DOI https://doi.org/

10.1055/s-0041-1741442. ISSN $0974-2727$.
(C) 2022. The Indian Association of Laboratory Physicians. All rights reserved.

This is an open access article published by Thieme under the terms of the Creative Commons Attribution-NonDerivative-NonCommercial-License, permitting copying and reproduction so long as the original work is given appropriate credit. Contents may not be used for commercial purposes, or adapted, remixed, transformed or built upon. (https://creativecommons.org/ licenses/by-nc-nd/4.0/)

Thieme Medical and Scientific Publishers Pvt. Ltd., A-12, 2nd Floor, Sector 2, Noida-201301 UP, India 


\section{Introduction}

Antiglobulin test is one of the commonest immunohematological investigations performed in the blood bank. The indirect antiglobulin test (IAT) detects the presence of free antibodies in the plasma as in prenatal testing of pregnant women as a part of hemolytic disease of fetus and newborn (HDFN) and in compatibility testing prior to blood transfusion. The direct antiglobulin test (DAT) detects immunoglobulin $\mathrm{G}(\mathrm{IgG})$ antibodies and complement components bound to red blood cell (RBC) antigens in vivo., ${ }^{1,2}$

The DAT is used primarily in the investigations of immune-mediated hemolysis such as hemolytic transfusion reactions, HDFN, autoimmune hemolytic anemia (AIHA), and drug-induced immune hemolysis. A positive DAT is almost always seen in association with AIHA and forms the hallmark of the diagnosis. ${ }^{3}$ Detection of red cell bound immunoglobulins and/or complement by DAT remains crucial serological assay in the diagnosis of AIHA. AIHA is characterized by increased red cell destruction due to autoantibodies directed against self-antigens on red cells. ${ }^{4}$

However, the mere existence of red cell bound immunoglobulins does not always indicate the presence of hemolysis. A combination of clinical and laboratory evidences of hemolysis is necessary to ascertain the diagnosis of autoimmune hemolysis. ${ }^{2}$ Studies have reported a high percentage of positive DAT in patients with a variety of acute illnesses. An incidence of positive DAT was observed in 1 to $15 \%$ of hospitalized patients without obvious features of hemolysis. ${ }^{5}$ Treatment with drugs, including intravenous immunoglobulins (IVIGs) and antithymocyte globulin is also known to be associated with positive DAT. ${ }^{6}$

Additionally, positive DAT, with no clear correlation with anemia, has been noted on red cells of patients with sickle cell disease, renal failure, and multiple myeloma. Thus, the interpretation of positive DAT should include patient's history, clinical data, and results of other laboratory investigations. ${ }^{2}$

The association of DAT with different clinical conditions has been described in several studies. ${ }^{1,2,5,7,8}$ However, none of these studies is from India. The correlation of DAT strength with presence of hemolysis has been reported only in a few studies published in the literature. The two Indian studies addressing this correlation are from the northern part of the country. ${ }^{4,9}$ None has been reported from the western region of the country. All the above-mentioned studies have individually characterized either clinical or serological features in case of a positive DAT or AIHA emphasizing the need of a comprehensive study that discusses both clinical and serological characteristics of positive DAT in detail.

Hence, a prospective observational study was undertaken to evaluate the clinical and serological correlation of positive DAT as the overall goal. The specific objectives were to categorize the clinical conditions associated with positive DAT, to estimate the presence of in vivo hemolysis in case of positive DAT with polyspecific and monospecific antisera and to correlate the strength of DAT positivity with the presence of hemolysis. The study also discusses the causes of false-positive, falsenegative tests, as well as preanalytical factors related to DAT.

\section{Materials and Methods}

\section{Study Setting and Ethical Approval}

It was a prospective observational study performed in the Department of Transfusion Medicine of a tertiary care center in western India. In our blood bank, we receive approximately 800 samples annually for DAT testing. Considering our institution to be a tertiary care hospital with superspecialty services, positive DAT results have been observed in variety of clinical conditions with or without hemolysis. The study was conducted from August 2016 to December 2017. Ethical approval was granted by the Institutional Ethics Committee (as per letter no IEC(I)/OUT/1022/2016 dated July 30, 2016).

\section{Sample Selection and Sample Size}

The study was performed on those samples that were sent by the clinicians to the Department of Transfusion Medicine for DAT investigation and were tested DAT positive. The DATpositive samples of neonates and the samples of patients who did not consent to participate were excluded from the study. The total sample size was 200 .

\section{Methodology}

The baseline DAT was performed on blood samples collected in ethylenediaminetetraacetic acid (EDTA) by tube technique using polyspecific antiglobulin reagent (Eryclone Antihuman Globulin, Tulip Diagnostics (P), Ltd, India) which contained antibodies to IgG and to C3d complement component. The test was performed using 3 to $5 \%$ suspension of red cells of the patient as per the manufacturer's instructions and Technical Manual, American Association of Blood Banks. ${ }^{10}$ The test was examined for agglutination. Test showing macroscopic agglutination was considered DAT positive. All the negative tests were confirmed to be true negative by using anti-D-coated DAT-positive control cells. The positive reactions were graded from $1+$ to $4+$ as per the Technical Manual. The patients with positive DAT were enrolled after their informed consent was obtained to participate in the study. Additional EDTA and nonanticoagulated samples, if required, were collected from patients for further laboratory work-up including monospecific DAT, hemoglobin, autologous control, and other biochemical parameters to assess hemolysis as mentioned later. DAT was further performed using monospecific antiglobulin antisera that contained antibodies to IgG (Erybank, Tulip Diagnostics (P), Ltd) and antibodies to C3d complement component (Eryclone, Tulip Diagnostics (P), Ltd) to detect whether red cells were coated with IgG or C3d or both. The tests were performed as per the manufacturer's instructions and the methods in the Technical Manual. ${ }^{10}$ The grades of positivity $(1+$ to $4+$ ) with both the monospecific antisera were recorded. Autologous control which tests the patient's serum with his or her own red cells was performed on all 200 positive DAT samples at $4^{\circ} \mathrm{C}, 22^{\circ} \mathrm{C}$, and $37^{\circ} \mathrm{C}$. The testing was performed by tube technique as per the Technical Manual. ${ }^{10}$

The clinical details were recorded including clinical examination, transfusion history, drug history, and other laboratory parameters. The patients were evaluated for the presence of 
Table 1 Disease categories of DAT-positive patients

\begin{tabular}{|l|l|l|}
\hline Clinical category & Number of patients & Percentage of total (\%) \\
\hline Autoimmune disorders & 75 & 37.5 \\
\hline Infectious diseases & 37 & 18.5 \\
\hline Malignancies (hematological and nonhematological) & 35 & 17.5 \\
\hline Nonmalignant hematological disorders & 25 & 12.5 \\
\hline Liver diseases (alcoholic and nonalcoholic hepatic cirrhosis) & 7 & 3.5 \\
\hline Cardiac diseases (valvular and coronary heart diseases) & 6 & 3 \\
\hline Miscellaneous diseases & 15 & 7.5 \\
\hline Total & 200 & 100 \\
\hline
\end{tabular}

Abbreviation: DAT, direct antiglobulin test.

hemolysis. Hemolysis was documented if at least three of the criteria were fulfilled: hemoglobin $(<9 \mathrm{~g} / \mathrm{dL})$, corrected reticulocyte count ( $>2 \%$ ), total serum bilirubin $(>2 \mathrm{mg} / \mathrm{dL}$ ), and lactate dehydrogenase $(\mathrm{LDH})(>500 \mathrm{IU} / \mathrm{mL})^{4,11}$

Total 200 samples that showed positivity with polyspecific antiglobulin reagent were further categorized based on the presence or absence of in vivo hemolysis. The clinical diagnoses of DAT-positive patients were reviewed and further grouped into broad clinical categories. Additionally, the presence or absence of hemolysis was assessed in each clinical category. The samples that tested positive with anti-IgG and/or anti-C3d monospecific antiglobulin reagents were also categorized into two groups based on whether hemolysis was present or absent in these samples. The presence or absence of hemolysis was further assessed for each grade (strength) of positivity with polyspecific and monospecific reagents.

\section{Statistical Analysis}

Descriptive statistics were applied to all the variables. Statistical analysis was done by IBM SPSS version 25. A p-value of less than 0.05 was considered statistically significant. Binomial logistic regression and Mann-Whitney $U$ test were applied to between the group analyses. For categorical variables, Fisher's exact test and relative risk were used. The qualitative data were expressed in numbers and percentages.

\section{Results}

Total 200 patients participated in the study. Out of them, 129 patients $(64.5 \%)$ were in the age group 20 to 50 years, while the overall range was 3 to 74 years. Among 200 patients, 82 were females and 118 were males.

The clinical diagnoses of the DAT-positive patients grouped into broad clinical categories are shown in - Table 1. Maximum number of patients, 75 of 200 , belonged to autoimmune disease group which constituted $37.5 \%$ of the total. Out of 75 patients, 31 had underlying systemic lupus erythematosus (SLE), while 18 had rheumatoid arthritis (RA). The chief infectious conditions associated with DAT positivity included tuberculosis (13/37) and hepatitis C infection (10/37). Acute myeloid leukemia and ovarian carcinoma were the commonest hematological and nonhematological malignancies, respectively. The nonmalignant hematological disorders associated with positive DAT were thalassemia major, idiopathic thrombocytopenic purpura, and thrombotic thrombocytopenic purpura.

Out of 200 DAT-positive patients, 98 (49\%) had in vivo hemolysis and 102 (51\%) did not have hemolysis. The presence or absence of in vivo hemolysis in different disease categories is shown in - Table 2. In 18 out of 44 patients of autoimmune diseases with hemolysis, diagnosis of SLE was established. Thus, 18 of total 31 SLE patients had in vivo hemolysis. Four patients with hemolysis had RA as their diagnosis. The diagnosis of primary AIHA was established in the remaining 22 of patients.

The commonest infectious disease associated with hemolysis was multidrug-resistant tuberculosis. Thalassemia major, chronic lymphocytic leukemia, and hepatocellular carcinoma were among the other principal clinical conditions associated with hemolysis.

- Table 3 shows the results of testing the samples with monospecific antiglobulin antisera and presence or absence of hemolysis in each category. Thus, 189 samples showed positivity with anti-IgG reagent and 88 samples showed positivity with anti-C3d reagent.

The grades of positivity with all the three categories of antiglobulin reagents were correlated with the presence or absence of hemolysis. - Tables 4-6 show the grades of positivity with polyspecific, monospecific anti-IgG, and monospecific anti-C3d reagents, respectively. The number of samples showing presence or absence of hemolysis for each grade of positivity is also shown in these tables. On binomial logistic regression analysis, it was found that there was statistically significant increase in the incidence of in vivo hemolysis with increasing grades of DAT positivity. This was observed with all the three reagents: polyspecific, monospecific anti-IgG, and monospecific anti-C3d, the $p$-values being $0.00,0.027$, and 0.001 respectively. For anti-C3, only those samples which showed positivity with both anti-IgG and anti-C3 were considered because the samples with only C3 positivity did not show hemolysis. 
Table 2 Disease categories with presence or absence of in vivo hemolysis

\begin{tabular}{|l|l|l|l|}
\hline Clinical category & Number of patients & $\begin{array}{l}\text { With in vivo } \\
\text { hemolysis }(\boldsymbol{n})\end{array}$ & $\begin{array}{l}\text { Without in vivo } \\
\text { hemolysis }(\boldsymbol{n})\end{array}$ \\
\hline Autoimmune disorders & 75 & 44 & 31 \\
\hline Infectious diseases & 37 & 11 & 26 \\
\hline Malignancy & 35 & 18 & 17 \\
\hline Nonmalignant hematological disorders & 25 & 18 & 7 \\
\hline Liver diseases & 7 & 4 & 3 \\
\hline Cardiac diseases & 6 & 1 & 5 \\
\hline Miscellaneous diseases & 15 & 2 & 13 \\
\hline Total & 200 & 98 & 102 \\
\hline
\end{tabular}

Table 3 DAT positivity with in vivo hemolysis

\begin{tabular}{|l|l|l|l|}
\hline DAT reagent used & Samples showing positive & \multicolumn{3}{|l|}{ In vivo hemolysis } \\
\cline { 3 - 4 } & result, $\boldsymbol{n}$ (\% of total) & $\begin{array}{l}\text { Present, } \boldsymbol{n} \text { (\% of all } \\
\text { hemolysis cases) }\end{array}$ & $\begin{array}{l}\text { Absent, } \mathbf{n} \text { (\% of all } \\
\text { non-hemolysis cases) }\end{array}$ \\
\hline Monospecific anti-IgG & & $44(39.3)$ & $68(66.7)$ \\
\hline Monospecific anti-IgG and monospecific anti-C3 & $77(38.5)$ & $54(60.7)$ & $23(22.5)$ \\
\hline Monospecific anti-C3 only & $11(5.5)$ & $00(00)$ & $11(10.8)$ \\
\hline Polyspecific reagent (total samples) & 200 & 98 & 102 \\
\hline
\end{tabular}

Abbreviations: DAT, direct antiglobulin test; IgG, immunoglobulin G.

Table 4 Grade of positive polyspecific DAT and presence of in vivo hemolysis

\begin{tabular}{|l|l|l|l|}
\hline $\begin{array}{l}\text { Grade of positive } \\
\text { DAT with polyspecific AHG }\end{array}$ & $\begin{array}{l}\text { Number of samples } \\
\text { (gradewise) }\end{array}$ & $\begin{array}{l}\text { Number of } \\
\text { samples in the grade } \\
\text { with in vivo hemolysis }\end{array}$ & $\begin{array}{l}\text { Number of samples } \\
\text { in the grade without } \\
\text { in vivo hemolysis (gradewise) }\end{array}$ \\
\hline $1+$ & 27 & 01 & 26 \\
\hline $2+$ & 98 & 33 & 65 \\
\hline $3+$ & 61 & 50 & 11 \\
\hline $4+$ & 14 & 14 & 00 \\
\hline Total & 200 & 98 & 102 \\
\hline
\end{tabular}

Abbreviations: AHG, antihuman globulin; DAT, direct antiglobulin test.

Table 5 Grade of positive anti-IgG DAT and presence of in vivo hemolysis

\begin{tabular}{|l|l|l|l|}
\hline $\begin{array}{l}\text { Grade of positive } \\
\text { DAT with anti-lgG AHG }\end{array}$ & $\begin{array}{l}\text { Number of } \\
\text { samples (gradewise) }\end{array}$ & $\begin{array}{l}\text { Number of } \\
\text { samples in the } \\
\text { grade with in vivo hemolysis }\end{array}$ & $\begin{array}{l}\text { Number of } \\
\text { samples in the grade } \\
\text { without in vivo hemolysis } \\
\text { (gradewise) }\end{array}$ \\
\hline $1+$ & 27 & 04 & 23 \\
\hline $2+$ & 118 & 57 & 61 \\
\hline $3+$ & 40 & 33 & 07 \\
\hline $4+$ & 04 & 04 & 00 \\
\hline Total & 189 & 98 & 91 \\
\hline
\end{tabular}

Abbreviations: AHG, antihuman globulin; DAT, direct antiglobulin test; IgG, immunoglobulin G. 
Table 6 Grade of positive anti-C3d DAT and presence of in vivo hemolysis

\begin{tabular}{|l|l|l|l|}
\hline $\begin{array}{l}\text { Grade of positive DAT } \\
\text { with anti-C3d AHG }\end{array}$ & $\begin{array}{l}\text { Number of } \\
\text { samples (gradewise) }\end{array}$ & $\begin{array}{l}\text { Number of } \\
\text { samples in the } \\
\text { grade with in vivo } \\
\text { hemolysis }\end{array}$ & $\begin{array}{l}\text { Number of samples } \\
\text { in the grade without } \\
\text { in vivo hemolysis (gradewise) }\end{array}$ \\
\hline $1+$ & 30 & 06 & 24 \\
\hline $2+$ & 36 & 26 & 10 \\
\hline $3+$ & 19 & 19 & 00 \\
\hline $4+$ & 03 & 03 & 00 \\
\hline Total & 88 & 54 & 34 \\
\hline
\end{tabular}

Abbreviations: AHG, antihuman globulin; DAT, direct antiglobulin test.

Table 7 Difference in mean grades between hemolysis and nonhemolysis groups with polyspecific, monospecific IgG, and monospecific C3d reagents

\begin{tabular}{|c|c|c|c|c|}
\hline Reagent & & $\begin{array}{l}\text { Hemolysis } \\
\text { present }\end{array}$ & $\begin{array}{l}\text { Hemolysis } \\
\text { absent }\end{array}$ & $\begin{array}{l}p \text {-Value of } \\
\text { difference in grade } \\
\text { between hemolysis } \\
\text { and nonhemolysis groups }\end{array}$ \\
\hline Polyspecific grade & Mean grade & 2.79 & 1.85 & $<0.001$ \\
\hline Anti-IgG grade & Mean grade & 2.38 & 1.63 & $<0.001$ \\
\hline Anti-C3d grade & Mean grade & 1.30 & 0.43 & $<0.001$ \\
\hline
\end{tabular}

Abbreviation: IgG, immunoglobulin G.

On Mann-Whitney $U$ test, there was statistically significant difference $(<0.001)$ in the scores of polyspecific grade, anti-IgG grade, and anti-C3d grade for hemolysis groups (-Table 7).

On investigating medication history, a history of IVIG was present in two patients with Guillain-Barre's syndrome. These two patients showed positive DAT with anti-IgG that was not associated with hemolysis. One patient with chronic myeloid leukemia with in vivo hemolysis was on antineoplastic drug-imatinib which is associated with drug-induced AIHA-drug-dependent antibody type. There was no specific drug history in other patients with in vivo hemolysis so as to associate it with drug-induced hemolysis.

History of RBC transfusion was present in 82 patients with positive DAT. Of them, 63 patients had in vivo hemolysis. Of these 63 patients, 29 had received red cell transfusions within the past 3 months, while 34 had received it before 3 months. Using Fisher's exact test, there was statistically significant association between transfusion history and presence of in vivo hemolysis irrespective of timing of transfusion $(p<0.00001)$.

Autocontrol was positive (grades $2+$ to $4+$ ) in 81 out of 98 cases with hemolysis which included all 44 cases of autoimmune diseases. The remaining 37 cases included 14 cases of nonmalignant hematological, 11 with malignant diseases, 9 cases of infectious diseases, and 3 cases with liver diseases. Forty-nine of 83 samples were DAT positive with both antiIgG and anti-C3d and 36 with anti-IgG alone. Of these 85 samples, 51 were positive only at $37^{\circ} \mathrm{C}, 29$ were positive at $37^{\circ} \mathrm{C}$ and $22^{\circ} \mathrm{C}$, and 5 were positive at all the three phases. Of 102 cases without hemolysis, 33 cases showed positive autocontrol (grades $1+$ to $3+$ ) at $4{ }^{\circ} \mathrm{C}$ and $22^{\circ} \mathrm{C}$. These included all 11 cases with DAT positivity only with antiC3d. The rest 22 samples showed positivity with both antiIgG and anti-C3d (15 cases) and with anti-IgG only ( 7 cases). The clinical conditions associated with these 33 cases were seen predominantly in autoimmune diseases (14), infectious diseases (9), malignant diseases (6), and other miscellaneous conditions (4).

\section{Discussion}

DAT is one of the most commonly performed investigations to rule out or rule in immune etiology as a part diagnostic work-up for anemia. It is a simple, quick, and inexpensive test. However, mere presence of positive DAT does not always indicate the presence of immune hemolysis. Hence, the present study was undertaken with the primary aim of evaluating the clinical and serological correlation of positive DAT.

The sample size was 200 DAT-positive patients. On grouping these patients into different disease conditions based on their clinical and laboratory features, it was found that 75/ 200 (37.5\%) patients belonged to autoimmune disease category (31 patients with SLE and 18 patients with RA). Earlier studies have shown DAT positivity in the presence of connective tissue disorders. ${ }^{12,13}$ In a study performed by Skare et al, DAT was positive in $12.8 \%$ of SLE patients. ${ }^{14} \mathrm{Xu}$ et al 
found that the presence of rheumatoid factor (RF) in plasma can lead to both false decreases and false increases in IAT and DAT. The interference effects are related to the RF content relative to the $\mathrm{IgG}$-sensitized $\mathrm{RBCs}{ }^{15}$ In the present study, hepatitis $C$ infection (16) and tuberculosis (11) were the commonest infectious diseases showing DAT positivity. Hepatitis $C$ infection is known to be associated with positive DAT. $^{16}$ In a study by Lai et al to evaluate DAT in human immunodeficiency virus (HIV)-infected individuals, hepatitis $C$ virus coinfection was shown to confer an increased risk for DAT positivity. ${ }^{17}$ The patients with hepatitis $\mathrm{C}$ infection in the present study were not on any treatment. High immunoglobulin levels have been shown to be associated with positive DAT. In patients with a higher level of IgG, the treatment of the concomitant disease resulted in normalization of the IgG level and led to a negative DAT test. ${ }^{18}$ Tuberculosis is known to be associated with polyclonal gammopathy. This could be the cause for positive DAT.

On further categorizing the DAT-positive samples based on the presence or absence of hemolysis, 98 (49\%) samples showed hemolysis and 102 (51\%) samples did not show hemolysis. Studies have shown positive DAT in hospitalized patients in the settings of acute illnesses who do not have overt signs of hemolysis or clearly evident etiology. $2,5,19$

In 18 out of 44 patients of autoimmune diseases with hemolysis, the diagnosis of SLE was established. Thus, 18 of total 31 SLE patients had in vivo hemolysis. Autoimmune hemolysis (defined as evidence of hemolysis, such as reticulocytosis, low haptoglobin, elevated indirect bilirubin or elevated LDH, and a positive DAT) is one of the classification criteria for SLE. ${ }^{20}$ Also, a study by Hanaoka et al has shown that a positive DAT in the absence of hemolytic anemia predicts high disease activity and poor renal response in SLE. ${ }^{21}$ In the study by Skare et al as mentioned earlier, DAT was positive in $12.8 \%$ of SLE studied sample and $54.3 \%$ of them had hemolytic anemia. ${ }^{14}$

Four patients with hemolysis had RA as their diagnosis. The diagnosis of primary AIHA was established in the remaining 22 of patients.

Six patients out of 11 with hemolysis in infectious disease category had hepatitis $C$ infection. Thus, out of 10 patients with hepatitis C, 6 had hemolysis. There are reports of DAT positivity and hemolytic anemia in association with hepatitis C infection. ${ }^{16,17,22}$ In vivo hemolysis of autoimmune etiology was detected in 4 out of 13 patients with tuberculosis. They all had disseminated tuberculosis. AIHA has been reported in cases of tuberculosis. ${ }^{23}$ Five patients with HIV positivity were shown to have positive DAT, and the association has been described in studies. ${ }^{24,25}$ Hemolysis seen in the remaining one case was due to plasmodium falciparum malaria. Out of 18 malignant conditions with hemolysis, 6 had chronic lymphocytic leukemia, 5 had Hodgkin's lymphoma, while 2 each had acute myeloid leukemia and prostatic cancer. The association of positive DAT with chronic lymphocytic leukemia and Hodgkin's disease has been described and hemolysis is known to occur in these conditions. ${ }^{26-28}$ Out of 18 patients with hemolysis, in the disease category of nonmalignant hematological disorders, 11 had transfusion-dependent thalassemia. Thalassemia itself being a hemolytic anemia with patients being on regular blood transfusions, they are prone to develop alloantibodies and autoantibodies to red cell antigen.

Monospecific antisera were used further to determine whether positive DAT was due to IgG or complement or both coating on the RBCs. In a study of patients with warm-type AIHA, the frequency of $\operatorname{IgG}$ alone ranged from 18 to $64 \%$; that of IgG and C3, from 34 to 65\%; and that of complement alone, from 10 to $33 \% .{ }^{29}$ In our study, none of the patients with DAT positivity due to C3 alone had in vivo hemolysis. Of 112 samples with only anti-IgG positivity, 44 (39.3\%) showed hemolysis and 68 (60.7\%) did not show hemolysis. Of 77 samples with both anti-IgG and anti-C3 positivity, 54 (70.1\%) showed hemolysis and 23 (29.9\%) did not show hemolysis. A study done by Zupanska et al revealed that the level of RBC sensitization with IgG was an important positive predictor of in vivo hemolysis. ${ }^{30}$

The increasing grades of positivity and presence of hemolysis are reported to have statistically significant association. The grades of reaction have been reported to be predictive of hemolysis. $^{8,31}$ In general, warm-type AIHA has been reported to have at least $3+$ grade. The amount of coated immunoglobulins determines the degree of hemolysis. In the present study, grades of DAT positivity were correlated with the presence or absence of hemolysis. The severity of hemolysis was not taken into consideration.

Various drugs have been known to be associated with positive DAT and in vivo hemolysis. ${ }^{2,6}$ In the present study, two patients with Guillain-Barre's syndrome had received IVIGs and the DAT was positive with anti-IgG reagent. However, they did not experience hemolysis. Positive DAT in patients on IVIG has been reported. ${ }^{32}$ Though most patients do not have clinically significant hemolysis, clinicians should be aware of this potentially serious complication. Antineoplastic drug imatinib that used in patient with chronic myeloid leukemia is known to be associated with drug-induced hemolysis and positive DAT. ${ }^{33}$

One case of acute myeloid leukemia had received out-ofgroup (O blood group) single donor platelet transfusion 3 days before the anti-IgG positive DAT test. There was in vivo hemolysis which is known if the donor has high-titer $\mathrm{ABO}$ hemagglutinins. ${ }^{34}$

Thalassemia major, connective tissue disorders, AIHA, and infectious diseases were the main clinical conditions that constituted the group of 63 patients with hemolysis who had history of red cell transfusions. Though a statistically significant association was found between history of transfusion and in vivo hemolysis, the anemia associated with these clinical conditions could be the reason for red cell transfusion requirement in these patients.

In our study, autocontrol was positive in 85 out of 98 cases with hemolysis. It was positive at $37^{\circ} \mathrm{C}$ in all cases with or without positivity at $4^{\circ} \mathrm{C}$ and $22^{\circ} \mathrm{C}$, indicating the presence of warm antibody in most cases responsible for in vivo hemolysis. Both warm and cold antibodies associated with hemolysis were observed in cases of infectious diseases and lymphoproliferative malignancies. These observations 
corroborate the earlier mentioned facts that SLE and other autoimmune diseases are associated with warm and mixed type of autoimmune hemolytic anemias. ${ }^{14,15}$ Chronic lymphocytic leukemia and hepatitis $C$ infection are known to be associated with cold agglutinin syndrome causing hemolysis. ${ }^{16,17}$ Development of autoantibody as well as alloantibody is known to occur in transfusion-dependent thalassemia major. ${ }^{35}$ In the patients who had received blood transfusion in the past 3 months, alloantibody directed against the transfused red cells is likely to be associated with positive DAT and positive autocontrol. In other patients with hemolysis with negative autocontrol, a drug-induced hemolysis needs to be investigated further. In patients without hemolysis, positive autocontrol was observed at lower temperatures indicating the presence of cold autoantibodies, not capable of causing hemolysis. In patients without hemolysis and negative autocontrol, conditions such as nonspecific autoagglutinins, IVIG treatment, polyclonal gammopathy, and high immunoglobulin levels need to be considered. ${ }^{1,2}$

Thus, autoantibodies were the major cause of positive DAT with or without hemolysis as in other studies., ${ }^{4,56}$ The autoantibody specificity was not determined in our study.

Out of 200 patients, 129 patients (64.5\%) were in the age group 20 to 50 years. There were 118 males and 82 females. However, 69 out of 75 cases of autoimmune disorders associated with positive DAT, and 41 of 44 cases of autoimmune disorders with hemolysis were females. These findings are consistent with other studies that autoimmune disorders are more common in females. ${ }^{36,37}$ Also, 66 of 75 cases of autoimmune disorders were found in age group above 40 years of age consistent with other studies.

DAT and autocontrol test were performed by the gold standard conventional tube technique. The technique is inexpensive and simple to perform and interpret. However, the results cannot be stored for later reference. In addition, the tube technique is fraught with false-positive and falsenegative reactions. Under-washing or under-centrifugation of the samples, failure to add antihuman globulin (AHG) reagent or addition of inactive AHG reagent, and delay in test performance are some of the causes of false-negative DAT. ${ }^{38}$ False-positive results may be due to nonspecific binding of the DAT reagents, over-centrifugation of sample, clotted sample, and reagent contamination. ${ }^{38}$ Higher level of $\operatorname{IgG}$ antibodies coated onto red cells of healthy individuals is an important confounding factor giving rise to positive DAT in these individuals. Patient-related preanalyical factors need considerations while interpreting DAT test result. Patients who are on IV medications containing colloid solutions lead to rouleaux formation that may result in false-positive DAT. False-negative DAT results may occur in instances of severe hemolysis where a great number of red cells are cleared so rapidly that only a few circulating sensitized RBCs are available for detection. In 5 to $10 \%$ of patients with immune hemolytic anemias, DAT may be negative if the number of IgG molecules bound to red cells are below the threshold limit of detection, or if hemolysis is mediated by $\operatorname{IgA}$ antibody. ${ }^{5}$
Considering all the aforementioned aspects associated with DAT, it can be said that DAT constitutes one of the first-line investigations in patients with hemolysis and in patients with history of transfusion to rule out underlying antibodies to ensure safer transfusion practices. It is important to investigate all DAT-positive samples further, to rule out underlying alloantibodies. A positive DAT must be interpreted in the light of medication history and clinical diagnosis. The pitfalls and preanalytical factors must be kept in mind.

\section{Limitations of the Study}

The specificity of autoantibodies could not be determined due to limited resource available. Sample inadequacy, severe anemia, and limited resources were the reasons for inability to perform elution/absorption studies required to evaluate and characterize alloantibodies.

\section{Conclusion}

Summarizing the results of this study, it can be concluded that there are various disease conditions which show positive DAT with or without hemolysis. So, it is important to clinically and serologically correlate positive DAT results to exclude false-positive reactions which may misguide the clinician. A pretransfusion DAT is recommended in multitransfused patients.

Note

The study was presented at a CME at Kokilaben Dhirubhai Ambani Hospital, Mumbai on August 29, 2019.

\section{Authors' Contribution}

A.K. contributed in writing the introduction and methodology sections. S.B. contributed in writing the results, discussion, and bibliography sections. J.S. contributed in reviewing the article.

\section{Ethical Approval}

The study was approved by the Institutional Ethics Committee Seth GS Medical College and KEM Hospital, Mumbai as per letter: IEC (I)/OUT/1022/16.

\section{Funding \\ None. \\ Conflict of Interest \\ None declared.}

\section{References}

1 Green REB, Hughes VC. The antiglobulin test. In: Denise Harmening, ed.Modern Blood Banking \& Transfusion Practices. Philadelphia: FA David Company; 2008:93-104

2 Leger RM. The positive direct antiglobulin test and immunemediated hemolysis. In: Roback JD, Grossman BJ, Harris T, Hillyer 
CD, eds. Technical Manual. 17th ed. Bethesda, MD: American Association of Blood Banks; 2011:437-462

3 Garratty G. Autoimmune hemolytic anemia. In: Garratty G, ed. Immunobiology of Transfusion Medicine. New York: Marcel Dekker; 1994:493-522

4 Das SS, Nityanand S, Chaudhary R. Clinical and serological characterization of autoimmune hemolytic anemia in a tertiary care hospital in North India. Ann Hematol 2009;88(08):727-732

5 Parker V, Tormey CA. The direct antiglobulin test: indications, interpretation, and pitfalls. Arch Pathol Lab Med 2017;141(02): 305-310

6 Arndt PA, Garratty G. The changing spectrum of drug-induced immune hemolytic anemia. Semin Hematol 2005;42(03): 137-144

7 Toy PT, Chin CA, Reid ME, Burns MA. Factors associated with positive direct antiglobulin tests in pretransfusion patients: a case-control study. Vox Sang 1985;49(03):215-220

8 Wikman A, Axdorph U, Gryfelt G, Gustafsson L, Björkholm M, Lundahl J. Characterization of red cell autoantibodies in consecutive DAT-positive patients with relation to in vivo haemolysis. Ann Hematol 2005;84(03):150-158

9 Chaudhary R, Das SS. Significance of quantitation of autoantibodies in the eluate of sensitized red cells in warm autoimmune hemolytic anemia. Laboratory Medicine 2009;40(09):531-534

10 Roback JD, ed. Technical Manual. 17th ed. Bethesda, MD: American Association of Blood Banks; 2011:905-906

11 Wheeler CA, Calhoun L, Blackall DP. Warm reactive autoantibodies: clinical and serologic correlations. Am J Clin Pathol 2004; 122(05):680-685

12 Mongan ES, Leddy JP, Atwater EC, Barnett EV. Direct antiglobulin (Coombs) reactions in patients with connective tissue diseases. Arthritis Rheum 1967;10(06):502-508

13 Kunkel HG. Immunological aspects of connective tissue disorders. Fed Proc 1964;23:623-626

14 Skare T, Picelli L, Dos Santos TAG, Nisihara R. Direct antiglobulin (Coombs) test in systemic lupus erythematosus patients. Clin Rheumatol 2017;36(09):2141-2144

$15 \mathrm{Xu} \mathrm{L}$, Li H, Yang S, et al. Interference in the indirect antiglobulin test and direct antiglobulin test from rheumatoid factor. J Int Med Res 2020;48(03):300060519892386

16 Elhajj II, Sharara AI, Taher AT. Chronic hepatitis C associated with Coombs-positive hemolytic anemia. Hematol J 2004;5(04):364-366

17 Lai M, d'Onofrio G, Visconti E, Tamburrini E, Cauda R, Leone G. Aetiological factors related to a positive direct antiglobulin test result in human immunodeficiency virus-infected patients. Vox Sang 2006;90(04):325-330

18 Bıçakçı Z, Öztürkmen S, Akyay A, Olcay L. False positive result of the direct antiglobulin test (DAT): the role of the elevated level of immunoglobulin G. Pediatr Hematol Oncol 2012;29(07):611-619

19 Freedman J. False-positive antiglobulin tests in healthy subjects and in hospital patients. J Clin Pathol 1979;32(10):1014-1018

20 Aringer M, Leuchten N, Johnson SR. New criteria for lupus. Curr Rheumatol Rep 2020;22(06):18

21 Hanaoka H, Iida H, Kiyokawa T, Takakuwa Y, Kawahata K. A positive direct Coombs' test in the absence of hemolytic anemia predicts high disease activity and poor renal response in systemic lupus erythematosus. Lupus 2018;27(14):2274-2278
22 Ramos-Casals M, García-Carrasco M, López-Medrano F, Trejo O, Forns X, López-Guillermo A, Muñoz C, Ingelmo M, Font J. Severe autoimmune cytopenias in treatment-naive hepatitis $C$ virus infection: clinical description of 35 cases. Medicine (Baltimore) 2003;82(02):87-96

23 Rathish D, Siribaddana S. Tuberculosis induced autoimmune haemolytic anaemia: a systematic review to find out common clinical presentations, investigation findings and the treatment options. Allergy Asthma Clin Immunol 2018;14:11

24 McGinniss MH, Macher AM, Rook AH, Alter HJ. Red cell autoantibodies in patients with acquired immune deficiency syndrome. Transfusion 1986;26(05):405-409

25 Zon LI, Arkin C, Groopman JE. Haematologic manifestations of the human immune deficiency virus (HIV). Br J Haematol 1987;66 (02):251-256

26 Abbas SA, Zeeshan R, Sultan S, Irfan SM. Direct Coombs test positivity in B-chronic lymphoid leukemia: a marker of advanced clinical disease. Asian Pac J Cancer Prev 2015;16(14): 6007-6010

27 Polliack A, Lugassy G. Autoimmunity and auto-immune syndromes associated with and preceding the development of lymphoproliferative disorders. Leukemia 1992;6(Suppl 4):152-154

28 Dearden C, Wade R, Else M, et al;UK National Cancer Research Institute (NCRI) Haematological Oncology Clinical Studies Group NCRI CLL Working Group. The prognostic significance of a positive direct antiglobulin test in chronic lymphocytic leukemia: a beneficial effect of the combination of fludarabine and cyclophosphamide on the incidence of hemolytic anemia. Blood 2008;111(04): $1820-1826$

29 Petz LD, Garratty G. Immune Hemolytic Anemias. 2nd ed. Philadelphia: Churchill-Livingstone; 2004

30 Zupanska B, Sokol RJ, Booker DJ, Stamps R. Erythrocyte autoantibodies, the monocyte monolayer assay and in vivo haemolysis. $\mathrm{Br}$ J Haematol 1993;84(01):144-150

31 Nathalang O, Sriwanitchrak P, Tubrod J, Kupatawintu P. Antibody elutions in Thai patients with a positive direct antiglobulin test. Blood Transfus 2011;9(03):306-310

32 Wilson JR, Bhoopalam H, Fisher M. Hemolytic anemia associated with intravenous immunoglobulin. Muscle Nerve 1997;20(09): 1142-1145

33 Quinley ED. Immunohematology: Principles and Practice. 3rd ed. Philadelphia, PA: Lippincott Williams \& Wilkins; 1998

34 Larsson LG, Welsh VJ, Ladd DJ. Acute intravascular hemolysis secondary to out-of-group platelet transfusion. Transfusion 2000; 40(08):902-906

35 Dhawan HK, Kumawat V, Marwaha N, et al. Alloimmunization and autoimmunization in transfusion dependent thalassemia major patients: study on 319 patients. Asian J Transfus Sci 2014;8(02): 84-88

36 Issit P. Serological diagnosis and characterization of causative antibody. In: Chaplin H Jr, ed. Methods in Hematology - Immune Hemolytic Anemia. United States: Churchill Livingston; 1985

37 Chaudhary RK, Das SS. Autoimmune hemolytic anemia: from lab to bedside. Asian J Transfus Sci 2014;8(01):5-12

38 Zantek ND, Koepsell SA, Tharp DR Jr, Cohn CS. The direct antiglobulin test: a critical step in the evaluation of hemolysis. Am J Hematol 2012;87(07):707-709 